\title{
Zahnarztpraxen spielen eine Schlüsselrolle für die Gesundheit
}

\author{
Die Amtszeit von Prof. Dr. Dr. Søren Jepsen als Präsident der European Federation of Periodonto- \\ logy (EFP) ist 2016 zu Ende gegangen. Ungeachtet des jährlichen Wechsels an der Spitze wird \\ Jepsen weiter im Vorstand des wissenschaftlichen Dachverbandes engagiert bleiben. In \\ Deutschland ist er Koordinator einer neuen Leitlinie der Arbeitsgemeinschaft der Wissenschaftli- \\ chen Medizinischen Fachgesellschaften (AWMF) zu "Diabetes und Parodontitis".
}

\begin{abstract}
DFZ: Seit 25 Jahren gibt es die EFP. Was bietet sie ihren Mitgliedern und der Zahnärzteschaft?

Jepsen: Die European Federation of Periodontology ist der Dachverband von 29 nationalen parodontologischen Fachgesellschaften, die insgesamt rund 15.000 Mitglieder haben. Übergeordnetes Ziel ist die Förderung der Parodontalgesundheit als Teil der Allgemeingesundheit durch Aus- und Fortbildung, Forschung und Kommunikation.
\end{abstract}

DFZ:Das Thema Prävention ist in der aktuellen Diskussion. Auch die EFP hat dies zu einem Thema gemacht. Was konnte die EFP hier beisteuern?

Jepsen: Prävention war das Leitthema unseres EFP Workshops Ende 2014. In vier Arbeitsgruppen wurden von 75 internationalen Experten Forschungsergebnisse zu diesem Thema systematisch begutachtet und aufbereitet. Sie betreffen die Grundlagen von Präventionskonzepten sowie die Behandlung von Gingivitis und periimplantärer Mukositis zur primären Prävention von Parodontitis und Periimplantitis. Außerdem beschäftigten wir uns mit den Konzepten, die bei zuvor erkrankten und bereits therapierten Patienten ein Wiederauftreten der Erkrankung verhindern sollen (sekundäre Prävention).

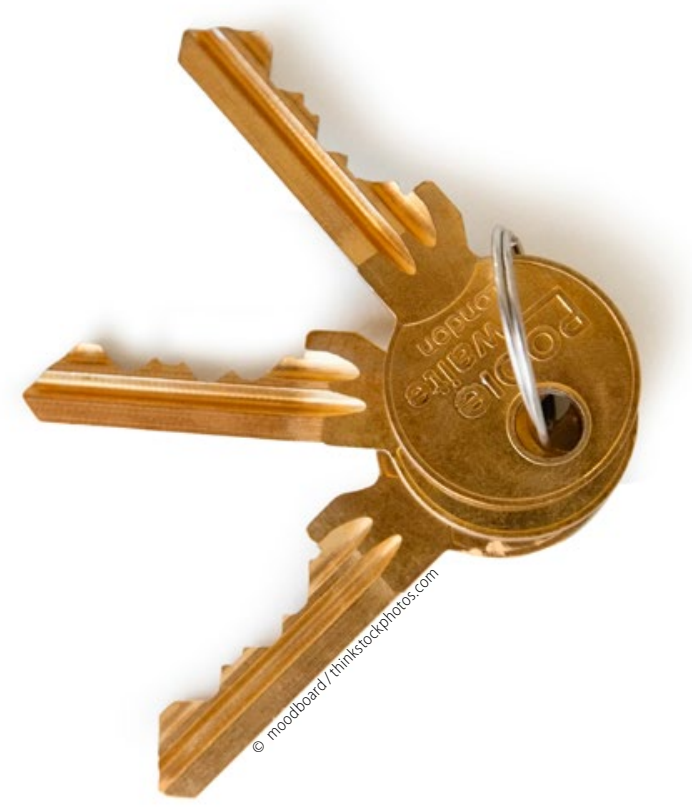

DFZ: Was sind dabei die leitenden Gedanken zum Thema Prävention? Jepsen: Wichtig ist uns, zu differenzieren zwischen der Primärprävention für Gesunde oder lediglich an Gingivitis Erkrankten und einer Sekundärprävention bei zuvor erkrankten, bereits therapierten Patienten. Die professionelle Zahnreinigung ist eine wichtige Maßnahme der Primärprävention. Wichtig ist, Risikofaktoren wie Rauchen und Diabetes zu berücksichtigen, darüber aufzuklären und Verhaltensänderungen in Richtung eines gesunden Lebensstils zu vermitteln.

DFZ: Sie sind Koordinator einer neuen AWMF-Leitlinie „Diabetes und Parodontitis". Heißt das, Sie räumen den Zahnärztinnen und Zahnärzten eine neue Rolle im Gesundheitssystem ein?

Jepsen: Wir sind überzeugt, dass Parodontologen und Zahnärzte eine wichtige Rolle spielen können, um Verhaltensänderungen und Gesundheitsförderung zu unterstützen. Es ist doch so, dass die Zahnarztpraxis eine Gesundheitseinrichtung ist, die von gesunden Menschen am häufigsten besucht wird. Darin liegt eine Chance, Krankheiten vorzubeugen oder sie zumindest im Frühstadium zu erkennen. Es geht um die Schlüsselrolle, die das zahnmedizinische Team bei der Verbesserung der Allgemeingesundheit spielen können. Mit der Leitlinie „Diabetes und Parodontitis“ wollen wir die Vernetzung von Arzt- und Zahnarztpraxen bei der Prävention und Behandlung der beiden Erkrankungen weiter vorantreiben. Die Leitlinie soll im Sommer 2017 beschlossen werden und eine wichtige Orientierungshilfe für die Praxis sein.

DFZ: Dazu wird es aber nicht reichen, die Zahnärzte und die Allgemeinärzte einzubinden. Die Aufklärung der Patienten wird damit umso wichtiger.

Jepsen: Wir sehen uns als EFP und als Deutsche Gesellschaft für Parodontologie in der Pflicht, Patienten aber auch gesundheitspolitische Entscheidungsträger aufzuklären. Deshalb gehen wir mit unserer Arbeit auch in die Öffentlichkeit und weisen auf die hohe Prävalenz der Parodontitis hin. Wir zeigen aber auch auf, wie bedeutsam Früherkennung ist und dass durch aktive und effektive Nachsorge Parodontitis verhindert oder auch schwere Parodontitis von qualifizierten Parodontologen gut behandelt werden kann. Wir fordern einen stärkeren Fokus auf Parodontologie in der studentischen Ausbildung. Natürlich sind dies langfristige Aufgaben. Wir haben mit dem europäischen Parodontologie-Tag ein wichtiges Zeichen gesetzt und Aufklärungsmaterialien erstellt, die an Patienten adressiert sind und welche die Praxen auch offensiv nutzen sollten.

red/DG PARO 